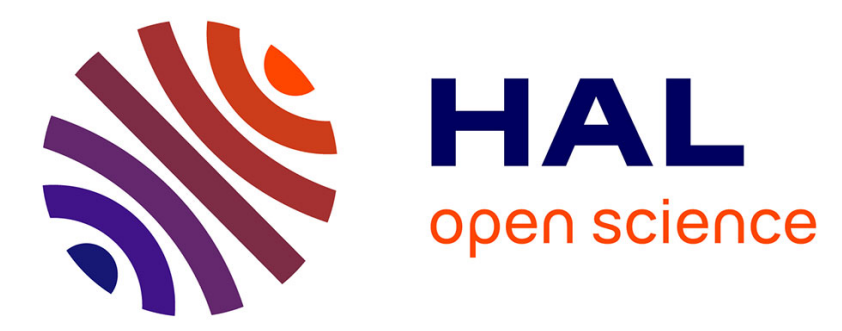

\title{
Calibration of the laser induced pressure pulse method when using a semiconducting electrode as the laser target
}

\author{
Peng Ma, Yewen Zhang, Stéphane Holé, Feihu Zheng, Zhenlian An
}

\section{- To cite this version:}

Peng Ma, Yewen Zhang, Stéphane Holé, Feihu Zheng, Zhenlian An. Calibration of the laser induced pressure pulse method when using a semiconducting electrode as the laser target. Measurement Science and Technology, 2016, 27 (2), pp.025003 10.1088/0957-0233/27/2/025003 . hal-01327247

\section{HAL Id: hal-01327247 https://hal.sorbonne-universite.fr/hal-01327247}

Submitted on 6 Jun 2016

HAL is a multi-disciplinary open access archive for the deposit and dissemination of scientific research documents, whether they are published or not. The documents may come from teaching and research institutions in France or abroad, or from public or private research centers.
L'archive ouverte pluridisciplinaire HAL, est destinée au dépôt et à la diffusion de documents scientifiques de niveau recherche, publiés ou non, émanant des établissements d'enseignement et de recherche français ou étrangers, des laboratoires publics ou privés. 


\title{
Calibration of the laser induced pressure pulse method when using semiconducting electrode as the laser target
}

\author{
Peng Ma ${ }^{2}$, Yewen Zhang ${ }^{1,2}$, Stéphane Holé ${ }^{1,3 *}$, Feihu Zheng ${ }^{1}$ and Zhenlian An $^{1}$ \\ ${ }^{1}$ Department of Electrical Engineering, Tongji University, Shanghai 201804, China \\ ${ }^{2}$ Shanghai Key Laboratory of Special Artificial Microstructure Materials and Technology, Department of Physics, \\ Tongji University, Shanghai 200092, China \\ ${ }^{3}$ Laboratoire de Physique et d'Étude des Matériaux (LPEM, UMR 8213), CNRS, Sorbonne Universités, UPMC \\ Univ Paris 06, PSL Research University, ESPCI-ParisTech, Paris 75005, France
}

Email: yewen.zhang@tongji.edu.cn; stephane.hole@tongji.edu.cn 


\begin{abstract}
The space charge distributions can directly be qualitatively estimated from the laser induced pressure pulse (LIPP) signal. To obtain precise quantitative space charge density, the calibration of LIPP method is necessary which requires an ideal measured signal. However, when a carbon loaded polymer electrode is used as the laser target, a long signal tail can be found. The cause of the signal tail is analyzed and two methods are proposed to correct the experimental signals. According to the calibration procedure introduced, space charge density is obtained respectively by using the two correction methods. Finally, the calculated results are compared and the validity of the calibration and correction methods is confirmed. The correction and calibration procedures proposed can be extended to piezoelectric induced pressure pulse (PIPP) and pulsed electroacoustic (PEA) methods.
\end{abstract}

Keywords: calibration procedure, correction method, space charge measurement, laser induced pressure pulse method, semiconducting electrode, insulating material 


\section{Introduction}

The usage of polymeric insulation in HVDC power transmission has a bright future due to the great environmental and economic advantages, compared with the paper-oil insulation [1]. However, the adverse effects of the accumulation of space charge under high direct current (dc) electric field, from electrical trees to partial discharges and breakdown [2-5], restrict the application of the polymeric insulation. Various direct and non-destructive techniques including the thermal $[6,7]$, pressure wave propagation (PWP) $[8,9]$ and pulsed electroacoustic (PEA) $[10,11]$ methods have been developed to study the impact of space charge accumulation on the electrical properties of the insulation. With the advantage of being with non-physical contact and generating a short rise time and large amplitude pressure pulse, the impact of a short laser pulse on a target was usually used as the pressure wave source in PWP method (i.e. laser induced pressure pulse method, LIPP) [12].

The principle of LIPP method has been well described [13] and the relevant technical problems encountered with LIPP method have also been summarized [14]. Theoretically, the space charge distributions can directly be qualitatively estimated from the measured signals in the LIPP experiments under short-circuit conditions. However, a calibration must be conducted if quantitative space charge distribution is needed. Usually, the calibration consists in measuring the signal amplitude for a known applied electric field, for instance the field in the sample free of charge [4]. In that latter situation, the amount of charges on the sample electrodes is precisely determined. Therefore, the signal amplitude can be used as the calibration coefficient for the next measurements in the case of a perfect pressure pulse. However, the LIPP signal can be distorted by a non-perfect pressure pulse. The pressure profile can indeed depend on the laser pulse energy, the target material and the physical conditions of the target [15]. A short depression following the pressure pulse was found when a $500 \mu \mathrm{m}$ thick aluminum (Al) plate or a $150 \mu \mathrm{m}$ thick quartz plate was used as the laser target in LIPP experiments [16, 17]. Signal tail or offset was also found with carbon loaded polymeric target thermally bonded to the sample, such as those used to approach a real high voltage cable structure [18]. In these cases, the distorted signals can be wrongly interpreted as charges. Therefore, a more complex calibration procedure has to be used [19], but it relies on difficult parameter adjustments and can add noise to the result.

In this paper, a novel procedure for charge density calibration with the LIPP method is described. This method is simple to implement and adds only little noise to the result. It is particularly well suited to measurements with polymeric target, such as carbon loaded ethylene-vinyl acetate copolymer, producing a long signal tail. After discussing the cause of the long signal tail, two methods are proposed to correct the measured signal. The results of both methods are compared in the last section before conclusion. 


\section{Calibration of LIPP method}

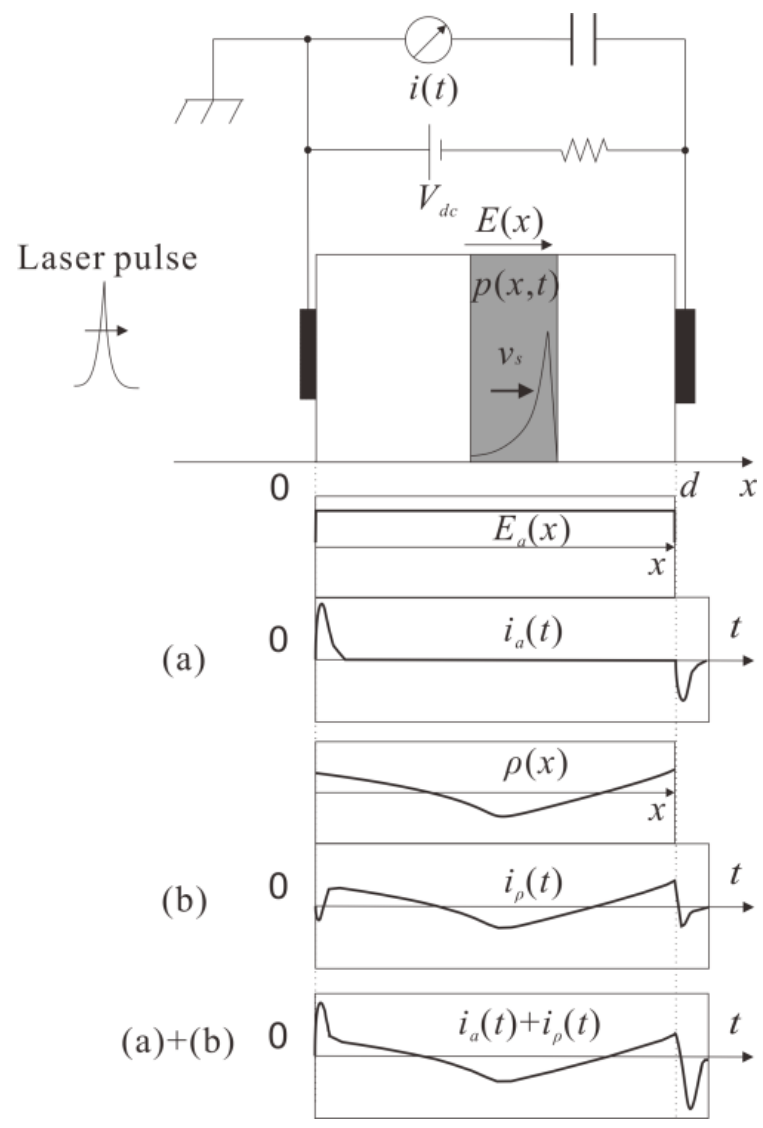

Figure 1. Principle of the pressure wave propagation method. (a) the current $i_{a}(t)$ induced by the pressure pulse due to the applied electric field $E_{a}(x)$, (b) the current $i_{\rho}(t)$ induced by the pressure pulse due to the space charge distribution $\rho(x)$ in the sample, (a)+(b) the current induced by the pressure pulse due to the combination of the applied electric field $E_{a}(x)$ and the space charge distribution $\rho(x)$.

The principle of the pressure wave propagation method is shown schematically in Figure 1. A short duration high power laser pulse is sent to the electrode which generates a pressure pulse by ablation [20]. The pressure pulse propagates at the velocity of sound through the sample. The material deformation produced by the propagation of the pressure pulse locally displaces the charges and changes the permittivity in the sample. Then a current $i(t)$ is induced in the measurement circuit which is an image of the charge distribution in short-circuit measurement conditions. The induced current is given by the relation

$$
i(t)=C_{0} \int_{0}^{d} B E(x) \frac{\partial p(x, t)}{\partial t} d x
$$

where $C_{0}$ is the sample capacitance at rest, $d$ is the thickness of sample, $E(x)$ is the electric field, $p(x, t)$ is the pressure pulse, $B$ depends on the characteristics of the sample. For homogeneous dielectric materials, parameter $B$ 
does not depend on positon $x$ and thus can be put outside the integral.

Integrating both sides of equation (1) over time and assuming that the extent of the pressure pulse is much shorter than the thickness of the sample, one has

$$
\int_{0}^{t} i\left(t^{\prime}\right) d t^{\prime}=C_{0} B \bar{E}\left(x=v_{s} t\right) \int_{0}^{d} p(x, t) d x
$$

where $\bar{E}(\mathrm{x})$ is the mean electric field at the scale of the pressure pulse extent and $v_{s}$ is the sound velocity.

Because dielectrics exhibit sound loss and sound dispersion, the amplitude of $p(x, t)$ decreases and the width of $p(x, t)$ increases during the propagation in the sample. The main action on the signal is the sound dispersion. Therefore, even if the profile of $p(x, t)$ is changing during the propagation, its integral $\int_{0}^{d} p(x, t) d x$ remains almost constant.

Then, the electric field can be obtained by

$$
\bar{E}\left(x=v_{s} t\right)=E_{0} \cdot \frac{\int_{0}^{t} i_{m}\left(t^{\prime}\right) d t^{\prime}}{\int_{0}^{t} i_{r}\left(t^{\prime}\right) d t^{\prime}}
$$

where $E_{0}$ is the reference electric field, $i_{r}$ is the reference signal obtained with $E_{0}, i_{m}$ is any other measured signals with the same sample, for instance in the presence of space charges. The reference electric field $E_{0}$ corresponds to the field in the sample free of charges. This electric field has to be sufficiently low as to prevent charges to be injected during its application.

If the pressure pulse profile is ideal and $\tau$ is its width at the half amplitude, one has

$$
\int_{0}^{t} i_{r}\left(t^{\prime}\right) d t^{\prime} \cong \int_{0}^{5 \tau} i_{r}\left(t^{\prime}\right) d t^{\prime}=\text { constant }
$$

According to the Possion formula in one dimension, the charge density is [21]

$$
\rho_{m}(x)=\varepsilon_{0} \varepsilon_{r} \frac{\partial E}{\partial x}=\frac{\varepsilon_{0} \varepsilon_{r}}{v_{s}} \cdot \frac{E_{0}}{\int_{0}^{5 \tau} i_{r}\left(t^{\prime}\right) d t^{\prime}} \cdot i_{m}\left(x / v_{s}\right)
$$

where $\varepsilon_{0}$ is the vacuum permittivity and $\varepsilon_{r}$ is the relative permittivity of the tested polymer insulator.

According to this calibration procedure, the space charge density can be obtained by simply multiplying the measured current by a constant. It needs to be pointed out that the measured current used in equation (5) must be an ideal signal. However, the practical measured current in the experiments always has various kinds of distortions. For instance, when carbon loaded ethylene-vinyl acetate copolymer (CB+EVA) is used as the laser target with the LIPP method, the generated pressure pulse does not return directly to zero but instead presents a long decreasing process that induces a long tail in the signal. Therefore Equation (4) no longer applies and the distorted signal can 
be confused with charges. Because $\mathrm{CB}+\mathrm{EVA}$ is used in many high voltage cable structures, it is very convenient to use it also as the laser target. Then some corrections on the measured signals must be carried out before they are used to calculate the space charge density from the calibration procedure.

\section{Signal interpretation and correction}

Because quantitative analysis is important for studying the behaviors of space charge in insulators, it is necessary to find ways to eliminate the distortions in the measured signals before applying the calibration procedure. In this section, the cause of the long signal tail is firstly discussed. Then two methods for correcting the signals are proposed.

\subsection{Cause of the long signal tail}

Two reasons can cause signal distortion: (1) the imperfect electrical impedance of the measuring circuit and (2) the undesirable pressure profile generated from the electrode. That latter reason is more physical, and then more complex to correct. We thus focus on that point in the following.

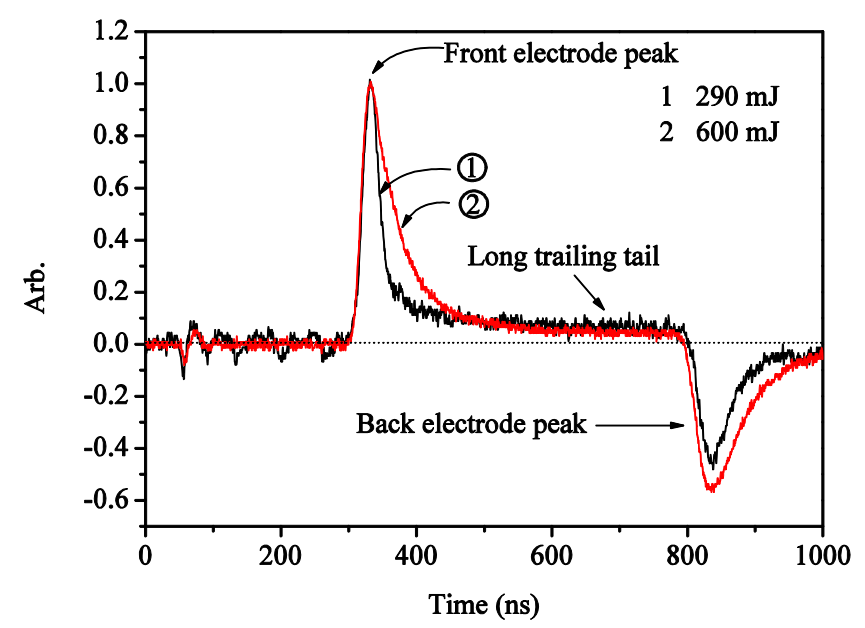

Figure 2. Comparison of normalized LIPP signals when using different laser pulse energies.

If the electrical impedance of the setup is perfect, for a sample without space charge under the reference electric field, the signal presents two peaks proportional to the practical pressure pulse at the interfaces between the insulator and front and back electrodes [19]. These two peaks are separated by the transit time of the pressure through the sample. In other words, the measured electrode peaks directly reflect the pressure profile. Both the laser pulse and the target material have an impact on the pressure profile produced as formerly discussed.

In a first measurement, a $1.0 \mathrm{~mm}$ thick low density polyethylene (LDPE) plate with $0.5 \mathrm{~mm}$ thick CB+EVA plate thermally bonded on both sides was placed in the testing chamber and measured with two laser pulse energies: $290 \mathrm{~mJ}$ or $600 \mathrm{~mJ}$. The sample was quickly measured under $5 \mathrm{kV}$ dc voltage under which no detectable 
charges have accumulated in the sample. For better comparison, the signals were normalized by the amplitude of the first electrode peak. The results are shown in Figure 2. It shows that a long signal tail is present in both cases though its relative amplitude decreases a little when the laser energy increases from $290 \mathrm{~mJ}$ to $600 \mathrm{~mJ}$.

In a second measurement, aluminum (Al) or $\mathrm{CB}+\mathrm{EVA}$ plate was respectively taken as the laser target when using the same laser pulse energy: $450 \mathrm{~mJ}$. Firstly, a sample consisting from left to right of a $3 \mathrm{~mm}$ thick $\mathrm{Al}$ plate, a $0.55 \mathrm{~mm}$ thick CB+EVA plate, a $1 \mathrm{~mm}$ thick LDPE plate and a $0.50 \mathrm{~mm}$ thick CB+EVA plate, was tested using the Al plate as the laser target. Secondly, the sample was tested using CB+EVA as the laser target by exchanging the positions of the $3 \mathrm{~mm}$ thick $\mathrm{Al}$ plate and $0.55 \mathrm{~mm}$ thick $\mathrm{CB}+\mathrm{EVA}$ plate. In that way there is the same materials, thus the same electric impedance, in the measurement circuit. The sample structures successively used are shown schematically in Figure 3. The materials are acoustically coupled together using a sufficiently thin layer of silicone oil to ensure good electrical contacts.

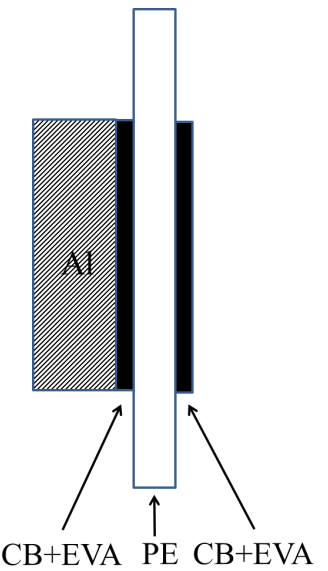

(a)

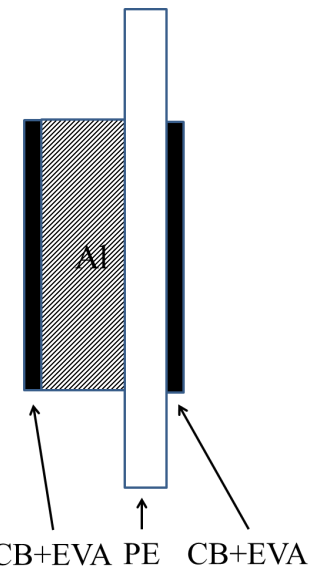

(b)

Figure 3. Schematic of the sample using $\mathrm{Al}$ or $\mathrm{CB}+\mathrm{EVA}$ as the laser target.

Figure 4 shows the normalized experimental results when $\mathrm{Al}$ or $\mathrm{CB}+\mathrm{EVA}$ electrodes are used as the laser target. It can be seen that the long signal tail is observed only when CB+EVA is used as the laser target. This indicates that the long tail in the signal is due to the pressure profile and not to the measurement circuit. The long tail may result from the relaxation (also known as resiliency) of CB+EVA material when the laser pulse hits the target surface. It has to be pointed out that the first peak width of the trace 1 is larger than that of trace 2 in Figure 4. The pressure generated by the laser ablation depends on the target material as above mentioned. It is not unreasonable to have different pulse widths when $\mathrm{Al}$ or $\mathrm{CB}+\mathrm{EVA}$ are separately used as the target. Though $\mathrm{Al}$ usually generates shorter pulses, a little alcohol was sputtered onto the surface to increase the pressure amplitude. It is known that it broadens the pulse width. In addition, the coupling between $\mathrm{Al}$ and $\mathrm{CB}+\mathrm{EVA}$ can also broaden 
the peak due to short duration standing wave in the coupling.

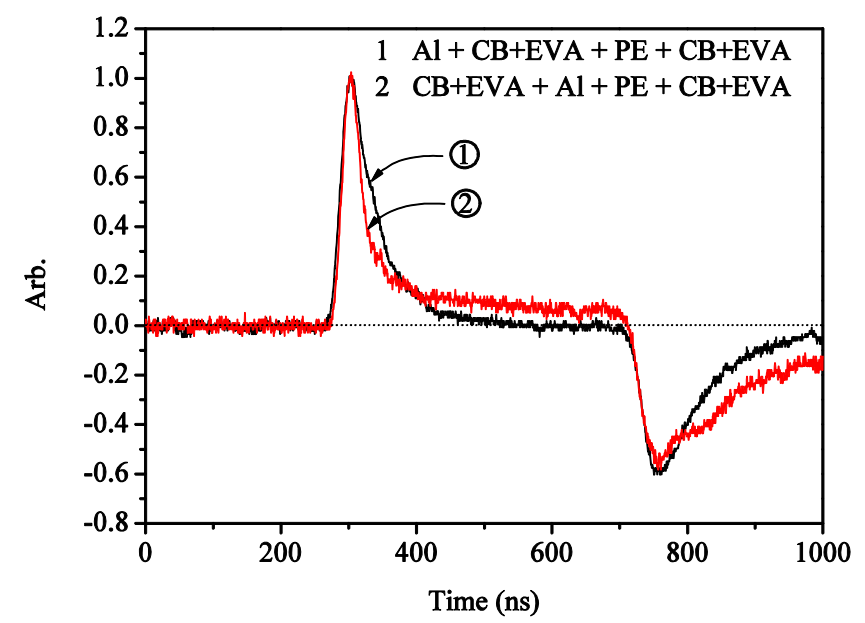

Figure 4. Comparison of the normalized LIPP signals when using different laser targets.

\subsection{Correction method}

The practical pressure pulse generated by the laser on the surface of the target can be seen as a convolution product between an ideal pressure pulse and a relaxation function $h(t)$. The practical pressure pulse induces the measured signal in short-circuit conditions. Likewise, the ideal pressure pulse would induce an ideal signal in the same conditions. Therefore, the measured signal can be also considered as a convolution product between an ideal signal and the relaxation function $h(t)$. In mathematical terms, one has

$$
i(t) * h(t)=i_{p}(t)
$$

where $*$ represents the operation of convolution, $i(t)$ is the signal from the ideal pressure pulse, $h(t)$ is the relaxation effect of the target material and $i_{p}(t)$ is the measured signal from the sample.

If $h(t)$ is known, the ideal current can be obtained through equation (6). It is usual with the PEA method that the first peak of the reference signal is directly used as $h(t)[11,22]$. However, this method adds noise and, in presence of a long signal tail, $h(t)$ must be truncated at least just before the second peak. This truncation deforms the spectrum of $h(t)$ which requires the application of complex filters. On the contrary, we propose two correction methods which connect the measured signal to the ideal signal, all calculations being made in time domain without windowing. Moreover, the methods add only little noise.

\subsubsection{Second order method}

Geometrical characteristics of the reference signal for the second order method are shown in Figure 5. $S_{m}, T_{p}$ and $t_{r}$ are respectively the amplitude of the maximum, Full-Width Half-Maximum (FWHM) and the $10 \%$ to $90 \%$ rise 
time of the peak. $S_{0}$ is the amplitude of the long signal tail at the end of the first peak in the signal. $t_{l}$ is the time duration of the peak and the left-hand and right-hand end-points of $t_{l}$ are taken respectively as the start of the first and second peaks. The geometrical characteristics are used to estimate the initial correction coefficients.

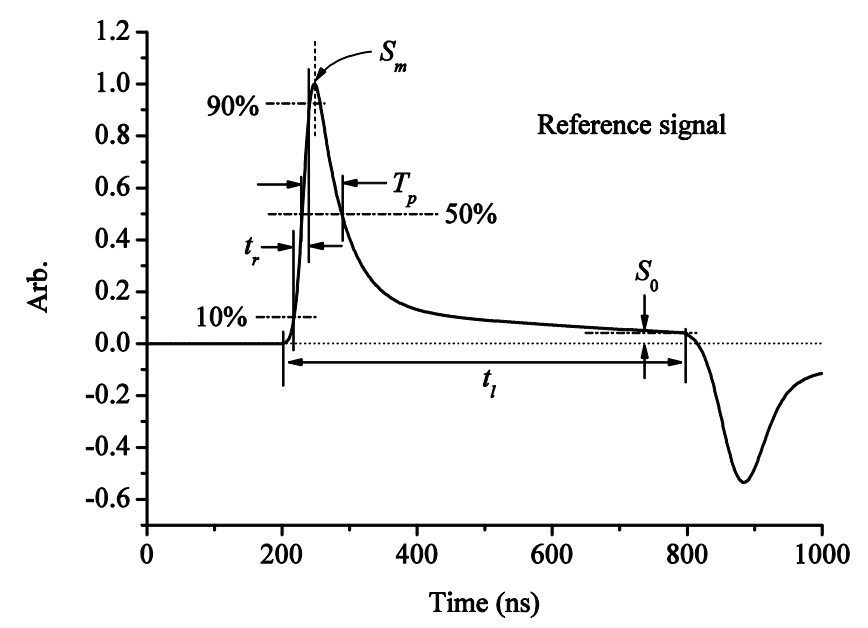

Figure 5. Geometrical characteristics of the reference signal for the correction coefficient estimation in the second order method.

An ideal current peak for the semiconducting CB+EVA electrode can be represented by the following empirical expression [23]

$$
i_{r}(t)=g(t) * \mathrm{e}^{-a t} U(t)=S_{g} e^{-\left(\frac{1.2 t}{t_{g}}\right)^{2}} * \mathrm{e}^{-a t} U(t)
$$

where $g(t)$ is a Gaussian function of amplitude $S_{g}$ and standard deviation $t_{g} / 1.2, a$ is a fitting coefficient which determines the falling portion of the ideal signal, and $U(t)$ is the Heaviside function.

If the response of the target was perfect, the laser induced current would be an ideal peak for the sample free of charge. In terms of linear systems, one can say that the impulse response of the target would be a Dirac function: $\delta(t)$. Due to resiliency, the response of the target includes a slow decaying process. Therefore, in addition to the ideal pulse, one obtains a slow decay and eventually an offset which mimics a very slow process compared to the measurement duration. The relaxation effect can always be expressed by a combination of an exponential function and an offset which can be represented by,

$$
h(t)=\left(\delta(t)+B \mathrm{e}^{-b t} U(t)\right) *(\delta(t)+C U(t))
$$

where $B$ is the amplitude of the long signal tail, $1 / b$ is its time constant and $C$ is the offset amplitude.

According to equation (6), the relationship between the ideal and measured signal can be expressed as

$$
i_{r}(t) *\left(\delta(t)+B \mathrm{e}^{-b t} U(t)\right) *(\delta(t)+C U(t))=i_{r p}(t)
$$


where $i_{r}(t)$ is the signal from the ideal pressure pulse and $i_{r p}(t)$ is the measured signal from the sample under the reference electric field.

In the Laplace space, one has

$$
I_{r}(s) \cdot\left(1+\frac{B}{s+b}\right) \cdot\left(1+\frac{C}{s}\right)=I_{r p}(s)
$$

Then the ideal signal can be expressed as

$$
I_{r}(s)=I_{r p}(s) \cdot \frac{s+b}{s+b+B} \cdot \frac{s}{s+C}
$$

The corresponding ideal signal in time domain is

$$
i_{r}(t)=i_{r p}(t) *\left(\delta(t)-X e^{-(b+B) t}-Y e^{-C t}\right)
$$

where $Y=C(\mathrm{~b}-C) /(b+B-C), \quad X=Y-B-C$.

The coefficients are adjusted by the Matlab fitting function fminunc according to Equation (7) and (9) with a classical quadratic optimization criterion. The optimization procedure is initialized by $B=0, b=1 /\left(2 t_{l}\right)$, $C=S_{0} /\left(1.4 t_{r} \cdot S_{m}\right), S_{g}=S_{m}, t_{g}=t_{r}$ and $a=2.2 / t_{f} \quad$ (see Figure 5). The correction results are shown in Figure 6. It can be noticed that the long signal tail has been successfully removed. The initial and the best fitted coefficients are listed in Table 1. The root mean square (RMS) error between the measured signal and fitted curve and the RMS of the measured signal noise can be compared to estimate the quality of the fit. It is found that the ratio between these two root mean squares is only 1.04. So it can be said that the difference between the measured signal and fitted curve is almost noise.

Table 1 Initial and best fitted coefficients used in the second order method.

\begin{tabular}{ccccccc}
\hline Initial & $S_{m}(\mathrm{~mA})$ & $t_{r}(\mathrm{~ns})$ & $a(1 / \mathrm{ns})$ & $B$ & $b(1 / \mathrm{ns})$ & $C$ \\
best fitted & 0.0156 & 22.4 & $1 / 13.6$ & 0 & $1 / 1334$ & 0.0010 \\
\hline
\end{tabular}




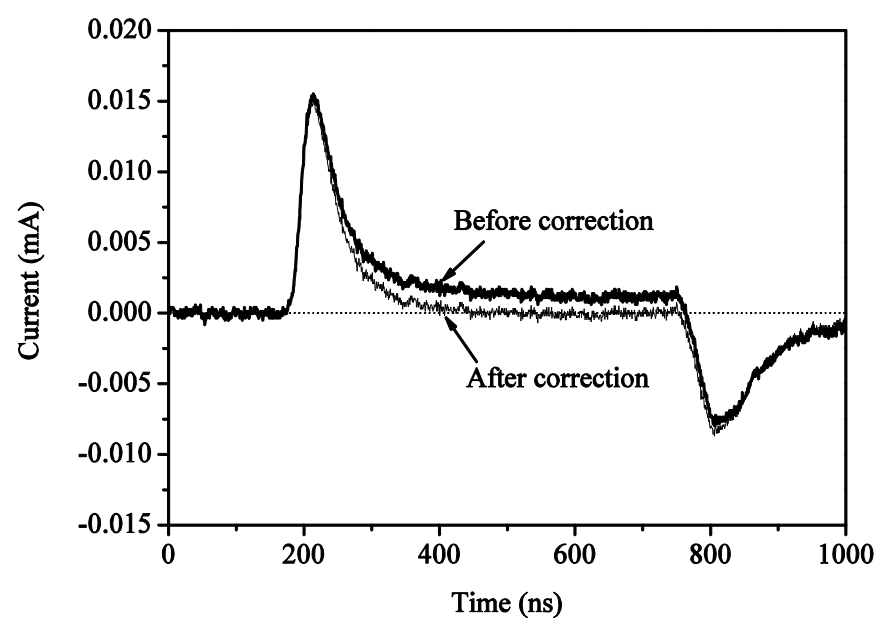

Figure 6. Reference signals before and after corrections by using the second order method.

\subsubsection{First order method}

Geometrical characteristics of the reference signal are shown in Figure 7. If $A_{m}$ and $t_{m}$ are respectively the amplitude and time position of the maximum of the peak, the long signal tail which is an exponential signal with a large time constant can be advantageously approximated by the linear function

$$
i_{l}=A_{r}-a_{r}\left(t-t_{m}\right)
$$

which amplitude is $A_{r}$ at time $t=t_{m}$ and which slope is $-a_{r}$. In that way, the parameters of the exponential response due to resiliency can be more easily estimated. We note $T_{p}$ the width of the pulse at the amplitude $A_{m} / 2+A_{r} / 4$. This amplitude almost corresponds to half the amplitude of the peak when taking into account the effect of resiliency.

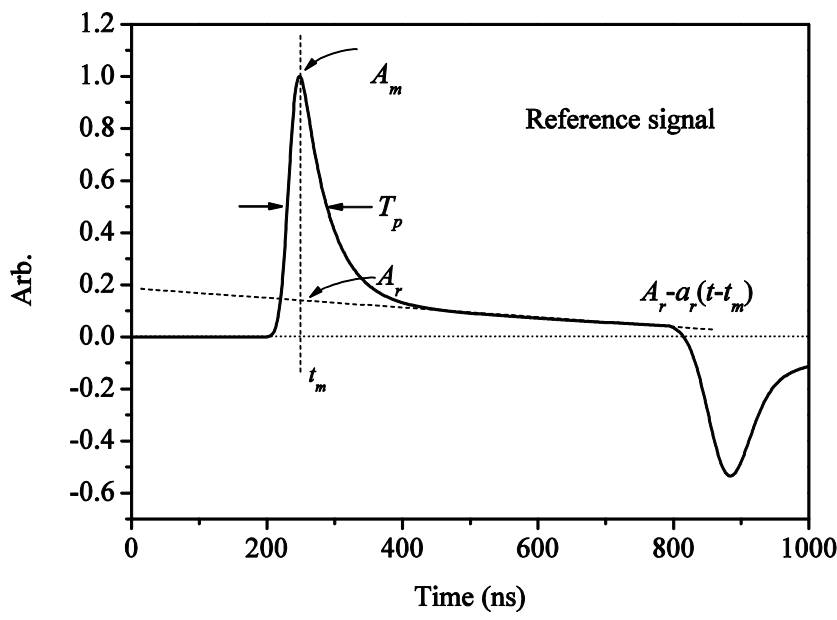

Figure 7. Geometrical characteristics of the reference signal for the correction coefficient estimation in the first order method. 
The long signal tail can be approximately expressed by an exponential expression. In terms of linear systems, one can say that the practical impulse response of the target is the sum of a Dirac and an exponential function,

$$
h(t)=\delta(t)+A e^{-a t} U(t)
$$

where $A$ is the amplitude of the resiliency effect, 1/a is its time constant and $U(t)$ is the Heaviside function [24].

Equation (6) can be rewritten as

$$
i_{r}(t) *\left(\delta(t)+A e^{-a t} U(t)\right)=i_{r p}(t)
$$

Which gives in the Laplace space,

$$
I_{r}(s) \cdot\left(1+\frac{A}{s+a}\right)=I_{r p}(s)
$$

Then

$$
I_{r}(s)=I_{r p}(s) \cdot \frac{s+a}{s+a+A}
$$

In the time domain, one has

$$
i_{r}(t)=i_{r p}(t) *\left[\delta(t)-A e^{-(a+A) t} U(t)\right]
$$

The coefficients are initialized by $A=2 A_{r} /\left[\left(2 A_{m}-A_{r}\right) T_{p}\right]$ and $a=a_{r} / A_{r}$ and optimized through the Matlab fitting function fminunc according to Equation (15) with a classical quadratic optimization criterion.

Figure 8 shows the uncorrected and corrected measured signals by using this method. The initial and adjusted coefficients are shown in Table 2. The correction is not very sensitive to coefficients $a$ and $A$ which have not to be precisely estimated. For example, a variation of $22 \%$ of coefficient $A$ induces here only a variation of $1.8 \%$ of the estimated electric field. Because the fit is only carried out on the tail of the measured signal, the RMS of the error between the measured signal and fitted curve is calculated on the signal after the first peak. Here we take the beginning of that signal section at three half peak widths from the first peak maximum and the end at the appearance of the second peak. Then the ratio between the RMS error and noise RMS is 1.08 . Here again the difference between the measured signal and fitted curve is almost noise.

Table 2. Initial and best fitted coefficients used in the first order method.

\begin{tabular}{cccc} 
initial & $A(1 / \mathrm{ns})$ & $a(1 / \mathrm{ns})$ & \\
best fitted & $1 / 537$ & $1 / 1452$ & \\
\hline & $1 / 688$ & $1 / 1452$ & \\
\hline
\end{tabular}




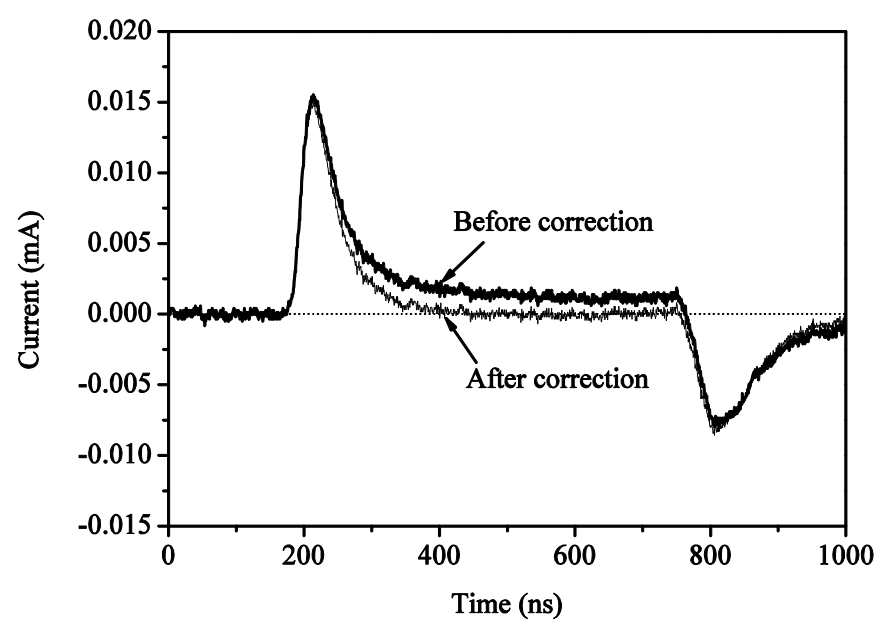

Figure 8. Reference signals before and after corrections by using the first order method.

\section{Experimental results}

\subsection{Procedure}

Experiments were carried out to test the validity of the calibration procedure and the correction methods. A LDPE plate with a thickness of $1.16 \mathrm{~mm}$ was used as the insulator and CB+EVA with a thickness of $0.6 \mathrm{~mm}$ was thermally bonded on both sides of the insulator.

Firstly, a signal under a dc voltage of $5.8 \mathrm{kV}$ is quickly measured (e.g. within a few minutes). This signal is used as the reference signal. Then, further signals under a dc voltage of $46.4 \mathrm{kV}$ are measured just after voltage on and $1.5 \mathrm{~h}$ after voltage on (e.g. $0 \mathrm{~min}, 1.5 \mathrm{~h}$ ). The applied dc voltage is then quickly reduced to zero and a final signal is immediately measured. The raw results are shown in Figure 9. It can be measured from the first peak of trace 1 or trace 2 that the FWHM of the electrode peak is about $59 \mathrm{~ns}$. There is an obvious discrepancy between the signals measured just before and just after voltage off (trace 3 and trace 4). One trace is shifted from the other between the peaks due to the long signal tail which results from the un-perfect pressure profile generated by laser ablation. Incorrect analyses may thus happen if the long signal tail is not considered. 


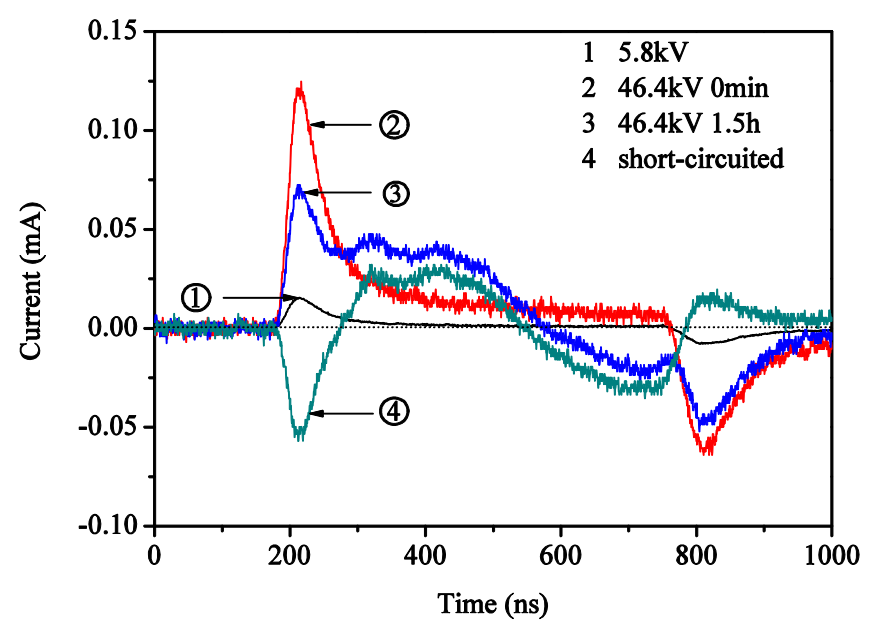

Figure 9. Evolution of LIPP signals in LDPE during $1.5 \mathrm{~h}$ under $46.4 \mathrm{kV}$.

\subsection{Corrected results}

The corrected results obtained with the two methods proposed in section 3 are shown in Figure 10. It can be seen that both methods process successfully the corrections and add almost no noise to the results. The resolution is improved from $59 \mathrm{~ns}$ to $49 \mathrm{~ns}$ by both correction methods. The long signal tail has been removed and the discrepancy between the signals at " $45.6 \mathrm{kV} 1.5 \mathrm{~h}$ " and at "short-circuited" becomes negligible.

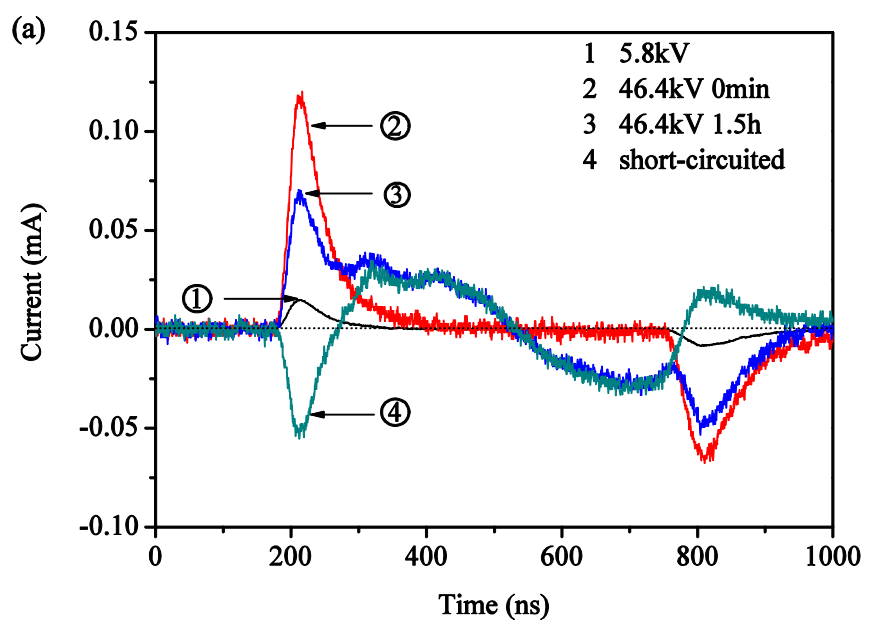




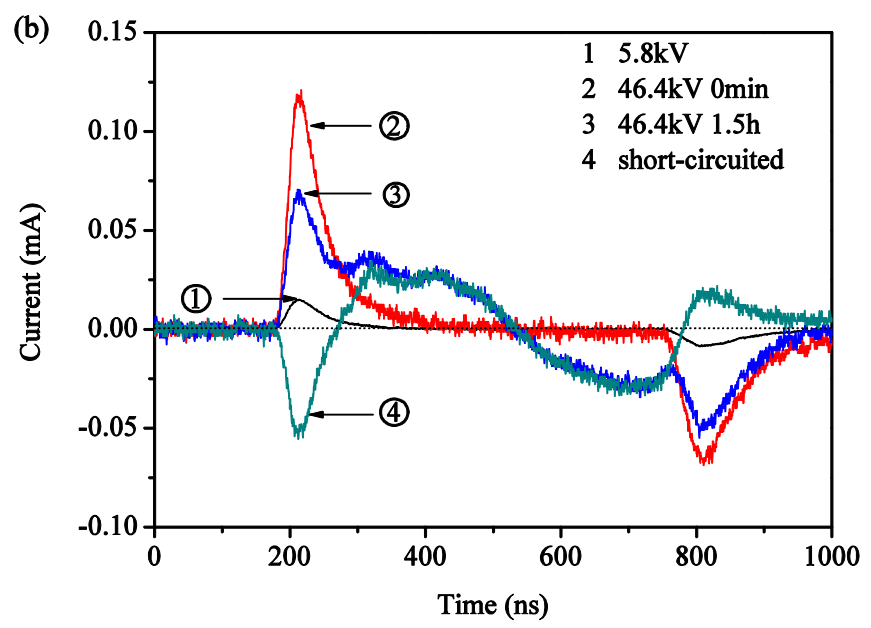

Figure 10. Corrected results from (a) the second order method and (b) the first order method.

\subsection{Calibration results}

Following the calibration procedure, the space charge distribution at $1.5 \mathrm{~h}$ under $46.4 \mathrm{kV}$ has been calculated with the two correction methods (Figure 11). It shows that the calculated space charge distributions are almost the same whatever the correction method being used.

The electric field and potential distributions in the sample are also shown in Figure 12. The estimated voltage value across the sample (Figure 12) is respectively $46.2 \mathrm{kV}$ and $45.6 \mathrm{kV}$ for second order and first order methods. The relative error of the potential for the two methods is respectively $0.4 \%$ and $1.5 \%$.

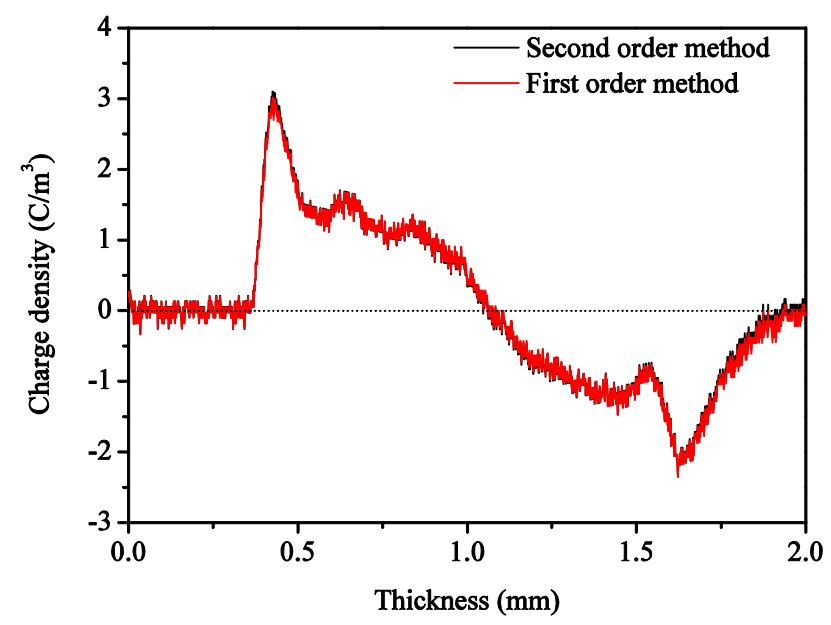

Figure 11. Comparison of the charge distributions from both correction methods (46.4 kV $1.5 \mathrm{~h})$. 


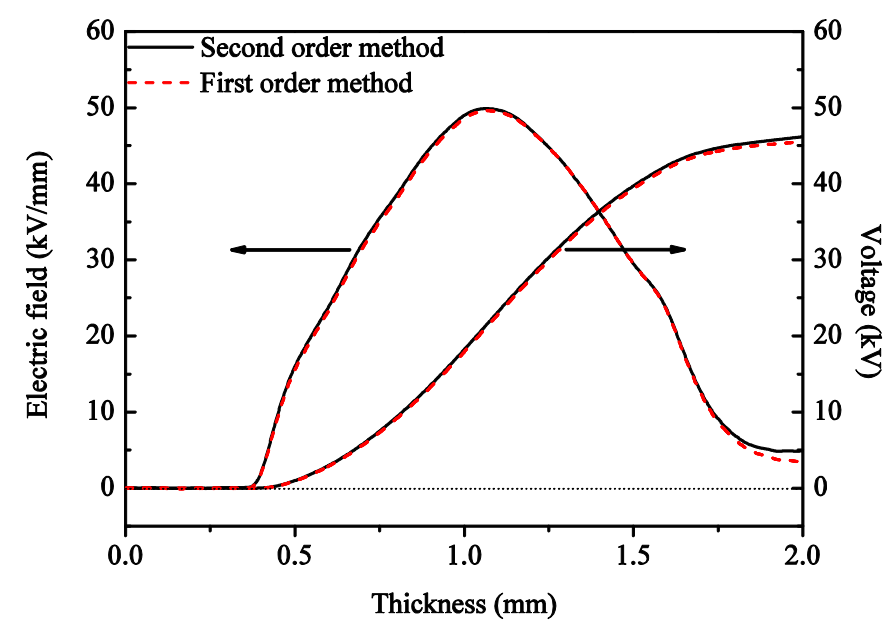

Figure 12. Comparison of the electric field and potential distributions for both correction methods $(46.4 \mathrm{kV}$ $1.5 \mathrm{~h})$.

\section{Conclusion}

The laser induced pressure pulse method (LIPP) is an important method for measuring the space charge distributions in polymeric insulator.

When carbon loaded ethylene-vinyl acetate copolymer ( $\mathrm{CB}+\mathrm{EVA})$ is used as the laser target, a long signal tail is observed in the signal which disturbs quantitative analysis. The undesirable pressure pulse profile was found to be responsible for the long signal tail. Two methods have been proposed to eliminate the long signal tail and both methods have successively been applied. The second order method completely describes the profile of the original signal whereas the first order method only describes the long signal tail. However, it is not always easy to find the best set of coefficients to succeed the second order method because of the number of parameters. The initial set of coefficients of the first order method is always good enough to conduct properly the correction. Therefore, it is more convenient to use the first order method to implement the correction in practice.

Almost the same space charge distributions are obtained through the calibration procedure once the measured signals are corrected. The calculated electric potential through the sample confirms the validity of both correction methods and calibration procedure. It can be pointed out that similar procedures for signal correction can be applied to other measurement techniques since both correction methods are based on signal analysis and not exclusively on the LIPP method. This could prevent signal windowing that imposes complex treatments in Fourier spectrum when applying conventional procedures

\section{Acknowledgments}

We gratefully acknowledge the financial support from the National Natural Science Foundation of China 
(NSFC Nos. 51477118 and 51477119) and PhD Programs Foundation of Ministry of Education of China (No. 20130072110046). 


\section{References}

[1] Sepulveda-Garcia M E, Martinez-Tarifa J M and Sanz-Feito J 2013 Electrical ageing markers for polyethylene insulation based on space charge accumulation and apparent mobility IEEE Trans. Dielectr. Electr. Insul. 20 2222-9

[2] Malec D 2007 Contribution to relationship between space charge injection and dielectric breakdown of low density polyethylene under a divergent field IEEE Trans. Dielectr. Electr. Insul. 14 502-7

[3] Dissado L A, Mazzanti G and Montanari G C 1997 The role of trapped space charges in the electrical aging of insulating materials IEEE Trans. Dielectr. Electr. Insul. 4 496-506

[4] Zhang Y, Lewiner J, Alquié C and Hampton N 1996 Evidence of strong correlation between space-charge buildup and breakdown in cable insulation IEEE Trans. Dielectr. Electr. Insul. 3 778-83

[5] Chen G, Tay T Y G, Davies A E, Tanaka Y and Takada T 2001 Electrodes and charge injection in low-density polyethylene IEEE Trans. Electr. Insul. 8 867-73

[6] Collins R E 1976 Analysis of spatial distribution of charges and dipoles in electrets by a transient heating technique J. Appl. Phys. 47 4804-8

[7] Lang S B and Das-Gupta D K 1986 Laser-intensity-modulation method: A technique for determination of spatial distributions of polarization and space charge in polymer electrets J. Appl. Phys. 59 2151-60

[8] Alquié C, Dreyfus G and Lewiner J 1981 Stress-wave probing of electric field distributions in dielectrics Phys. Rev. Lett. 47 1483-7

[9] Sessler G M, West J E and Gerhard G 1982 High-resolution laser-pulse method for measuring charge distributions in dielectrics Phys. Rev. Lett. 48 563-6

[10] Maeno T, Futami T, Kushibe H, Takada T and Cooke C M 1988 Measurement of spatial charge distribution in thick dielectrics using the pulsed electroacoustic method IEEE Trans. Electr. Insul. 23 433-9

[11] Li Y, Yasuda M and Takada T 1994 Pulsed electroacoustic method for measurement of accumulation in solid dielectrics IEEE Trans. Dielectr. Electr. Insul. 1 188-95

[12] Lewiner J, Holé S and Ditchi T 2005 Pressure wave propagation methods: A rich history and a bright future IEEE Trans. Dielectr. Electr. Insul. 12 114-26

[13] Holé S and Ditchi T 2003 Non-destructive methods for space charge distribution measurements: What are the differences? IEEE Trans. Dielectr. Electr. Insul. 10 670-7

[14] Malec D 2000 Technical problems encountered with the laser induced pressure pulse method in studies of high voltage cable insulators Meas. Sci. Technol. 11 76-80

[15] Alquié C, Lewiner J and Dreyfus G 1983 Analysis of laser induced acoustic pulse probing of distributions in dielectrics Journal de physique. Lettres 44 171-8

[16] Cals M P, Marque J P and Alquié C 1992 Application of the pressure wave propagation method to the study of interfacial effects in e - irradiated polymer films J. Appl. Phys. 72 1940-51

[17] Gerhard-Multhaupt R, Sessler G M, West J E, Holdik K, Haardt M and Eisenmenger W 1984 Investigation of piezoelectricity distributions in poly(vinylidene fluoride) by means of quartz- or laser-generated pressure pulses J. Appl. Phys. 55 2769-75

[18] An Z, Liu C, Chen X, Zheng F and Zhang Y 2013 Influence of oxygen impurity in fluorinating mixtures on charge blocking properties of fluorinated surface layer of polyethylene IEEE Trans. Dielectr. Electr. Insul. 20 $303-10$

[19] Ditchi T, Alquié C and Lewiner J 1993 Broadband determination of ultrasonic attenuation and phase velocity in insulating materials J. Acoust. Soc. Am. 94 3061-6

[20] O'Keefe J D and Skeen C H 1972 Laser-induced stress-wave and impulse augmentation Appl. Phys. Lett. 21 
464-6

[21] Holé S, Dissado L A, Fothergill J C and Lewiner J 2008 Direct analysis of heterogeneous insulators with the PWP and the PEA methods 13th ISE (Tokyo, Japan) pp C112

[22] Chen G, Chong Y L and Fu M 2006 Calibration of the pulsed electroacoustic technique in the presence of trapped charge Meas. Sci. and Technol. 17 1974-80

[23] Hu L, Zhang Y and Zheng F 2005 Novel numerical methods for measuring distributions of space charge and electric field in solid dielectrics with deconvolution algorithm IEEE Trans. Electr. Insul. 12 809-14

[24] Holé S, Perrillat D and Blanc H 2013 Effect of non-uniformly distributed talc fillers in polypropylene under high voltage ICSD'13 (Bologna, Italy) pp 460-3 


\section{Figure captions}

Figure 1. Principle of the pressure wave propagation method. (a) the current $i_{a}(t)$ induced by the pressure pulse due to the applied electric field $E_{a}(x)$, (b) the current $i_{\rho}(t)$ induced by the pressure pulse due to the space charge distribution $\rho(x)$ in the sample, (a)+(b) the current induced by the pressure pulse due to the combination of the applied electric field $E_{a}(x)$ and the space charge distribution $\rho(x)$.

Figure 2. Comparison of normalized LIPP signals when using different laser pulse energies.

Figure 3. Schematic of the sample using $\mathrm{Al}$ or $\mathrm{CB}+\mathrm{EVA}$ as the laser target.

Figure 4. Comparison of the normalized LIPP signals when using different laser targets.

Figure 5. Geometrical characteristics of the reference signal for the correction coefficient estimation in the second order method.

Figure 6. Reference signals before and after corrections by using the second order method.

Figure 7. Geometrical characteristics of the reference signal for the correction coefficient estimation in the first order method.

Figure 8. Reference signals before and after corrections by using the first order method.

Figure 9. Evolution of LIPP signals in LDPE during $1.5 \mathrm{~h}$ under $46.4 \mathrm{kV}$.

Figure 10. Corrected results from (a) the second order method and (b) the first order method.

Figure 11. Comparison of the charge distributions from both correction methods ( $46.4 \mathrm{kV} 1.5 \mathrm{~h}$ ).

Figure 12. Comparison of the electric field and potential distributions for both correction methods $(46.4 \mathrm{kV}$ $1.5 \mathrm{~h})$. 
Table 1 Initial and best fitted coefficients used in the second order method.

\begin{tabular}{ccccccc}
\hline initial & $S_{m}(\mathrm{~mA})$ & $t_{r}(\mathrm{~ns})$ & $a(1 / \mathrm{ns})$ & $B$ & $b(1 / \mathrm{ns})$ & $C$ \\
best fitted & 0.0156 & 22.4 & $1 / 13.6$ & 0 & $1 / 1334$ & 0.0010 \\
\hline
\end{tabular}

Table 2. Initial and best fitted coefficients used in the first order method.

\begin{tabular}{|c|c|c|}
\hline values & $A(1 / \mathrm{ns})$ & $a(1 / \mathrm{ns})$ \\
\hline initial & $1 / 537$ & $1 / 1452$ \\
\hline best fitted & $1 / 688$ & $1 / 1452$ \\
\hline
\end{tabular}


Figure 1.

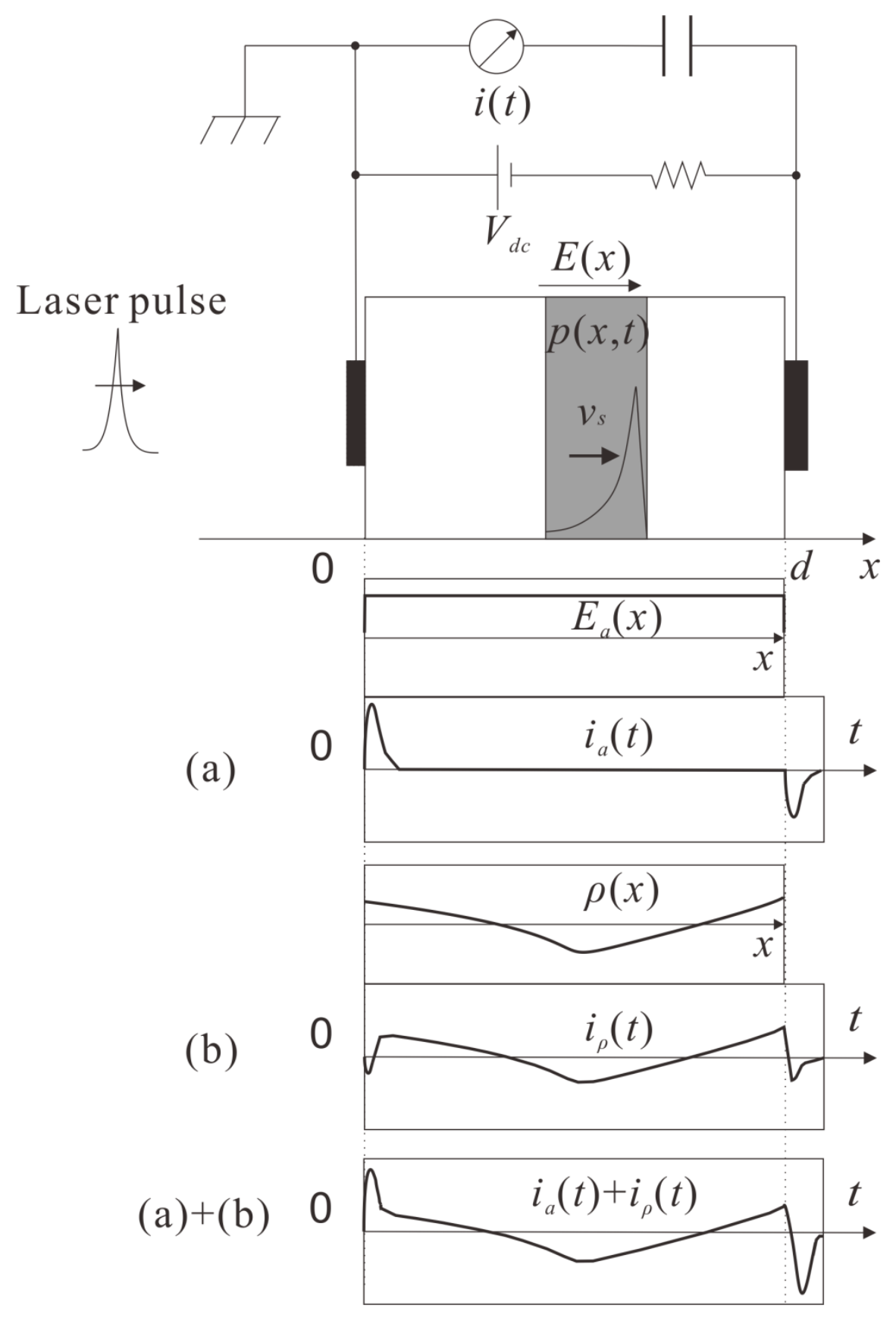




\section{Figure 2.}

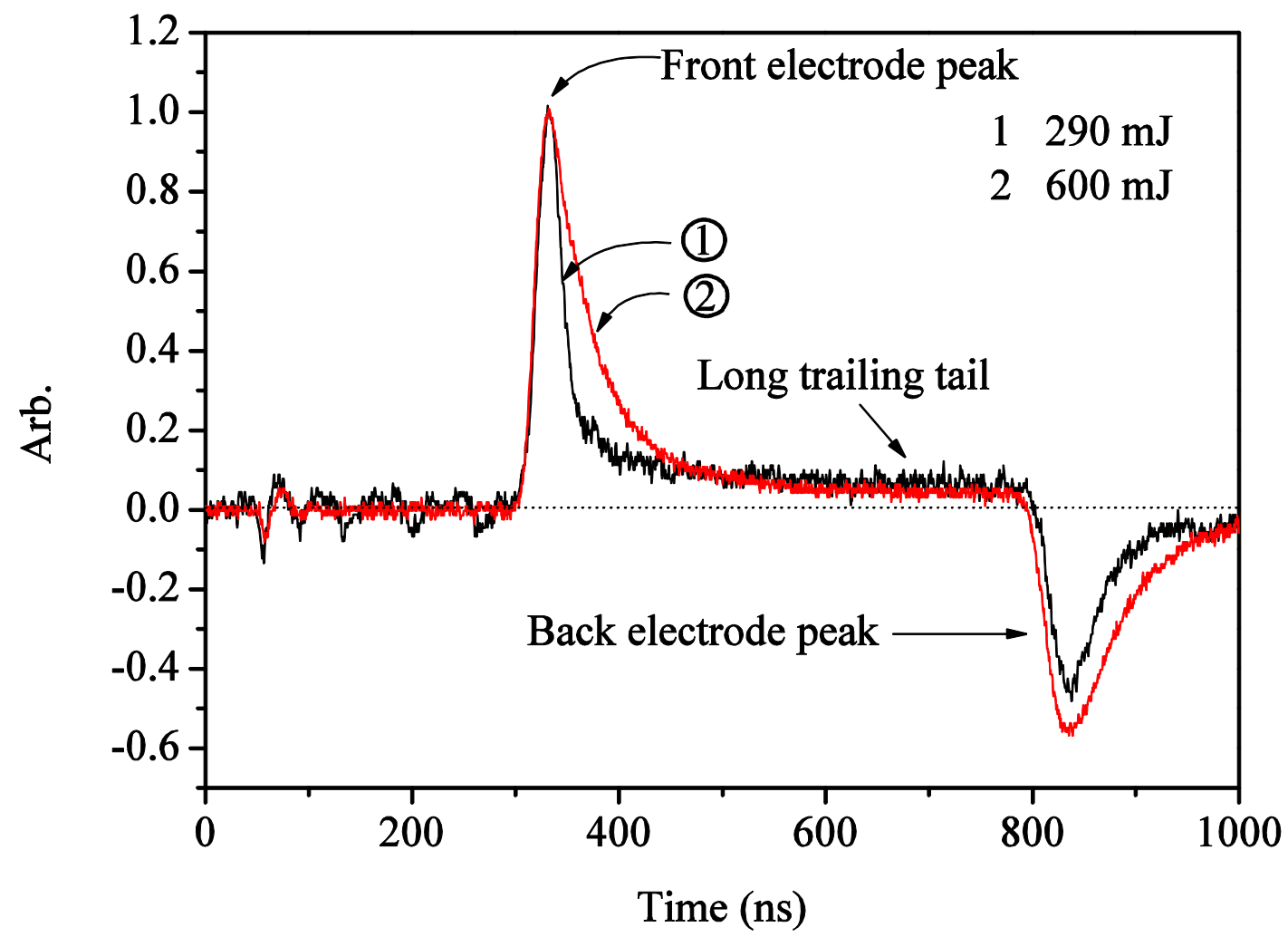


Figure 3.

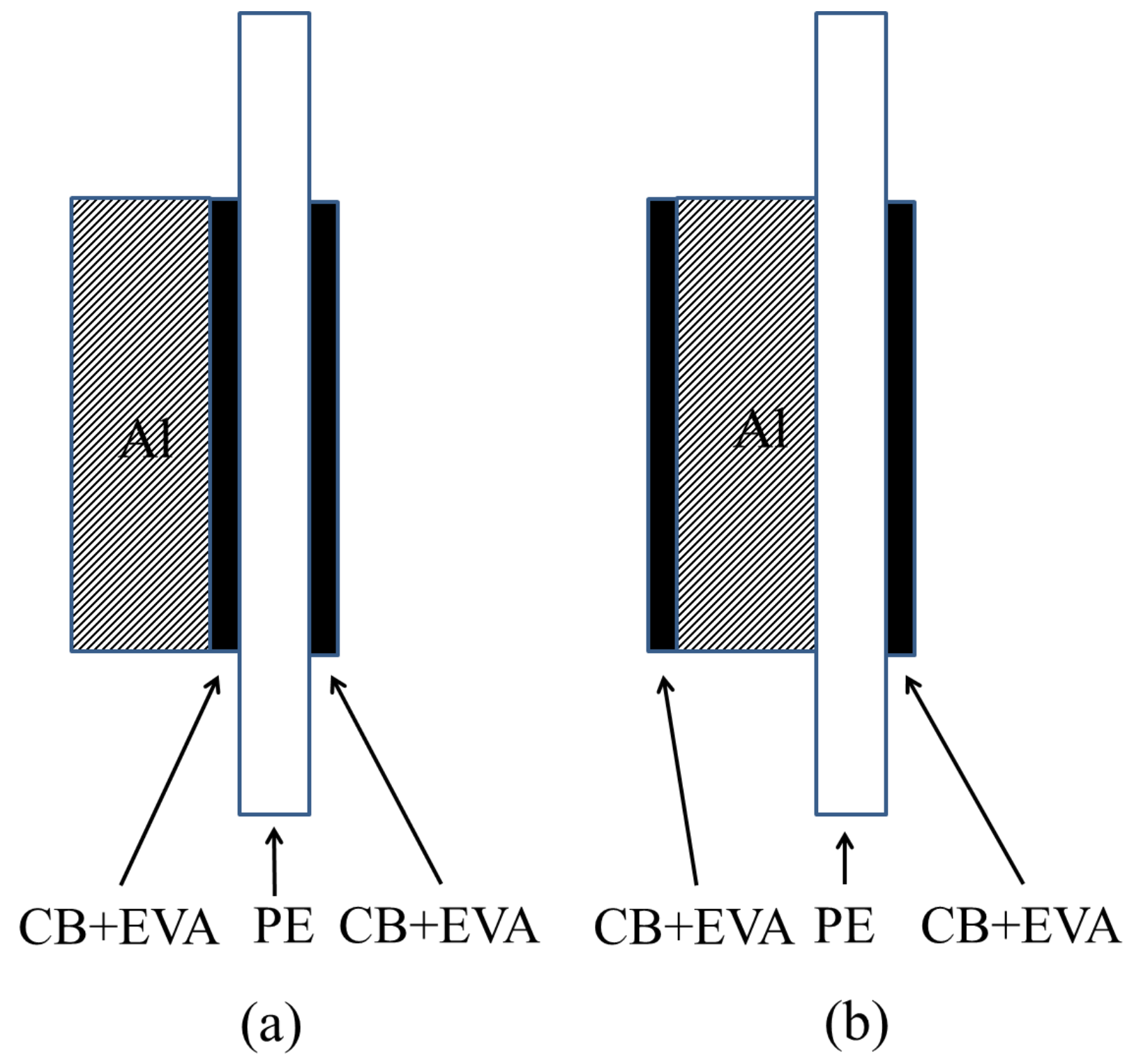




\section{Figure 4.}

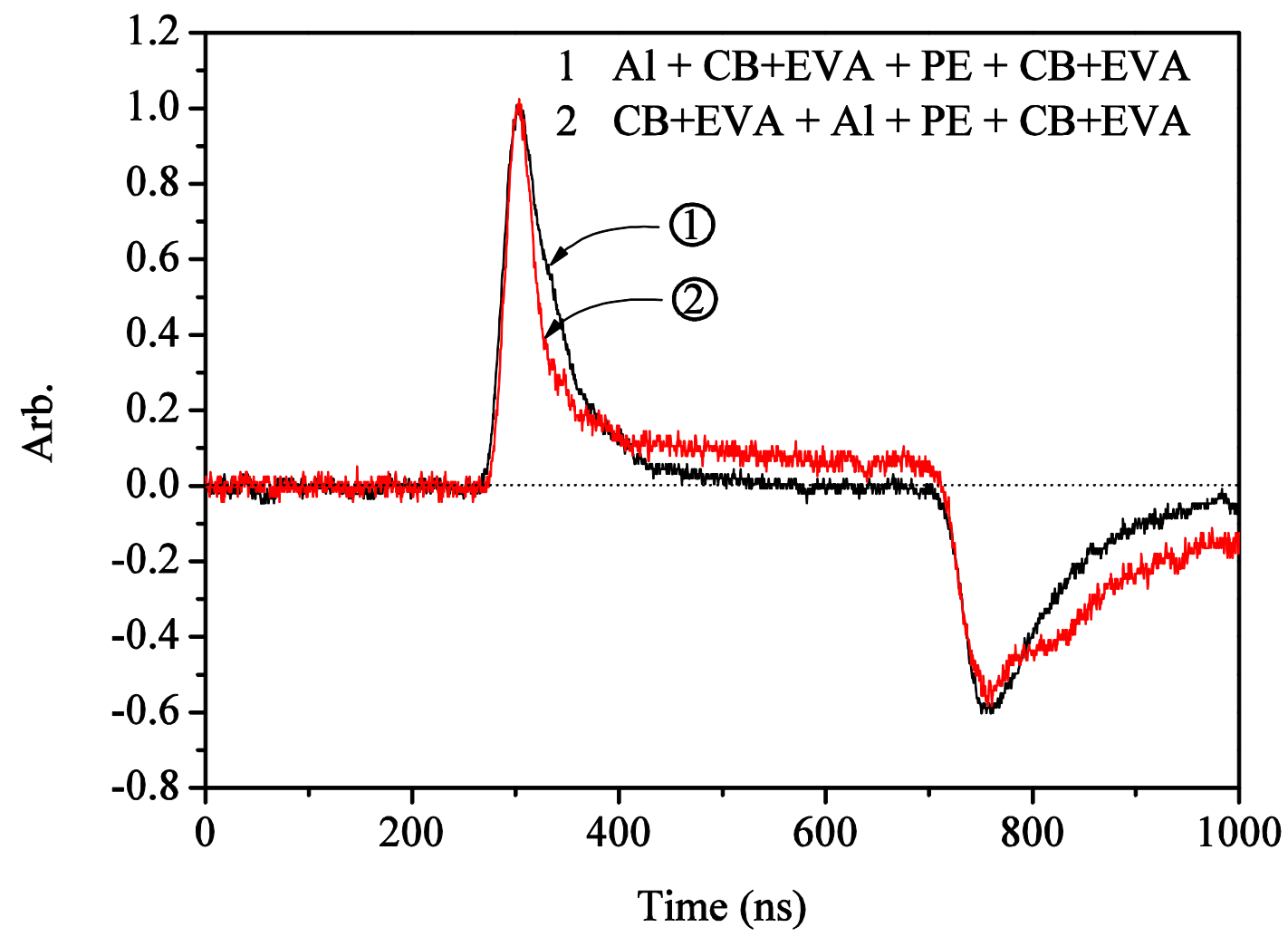




\section{Figure 5.}

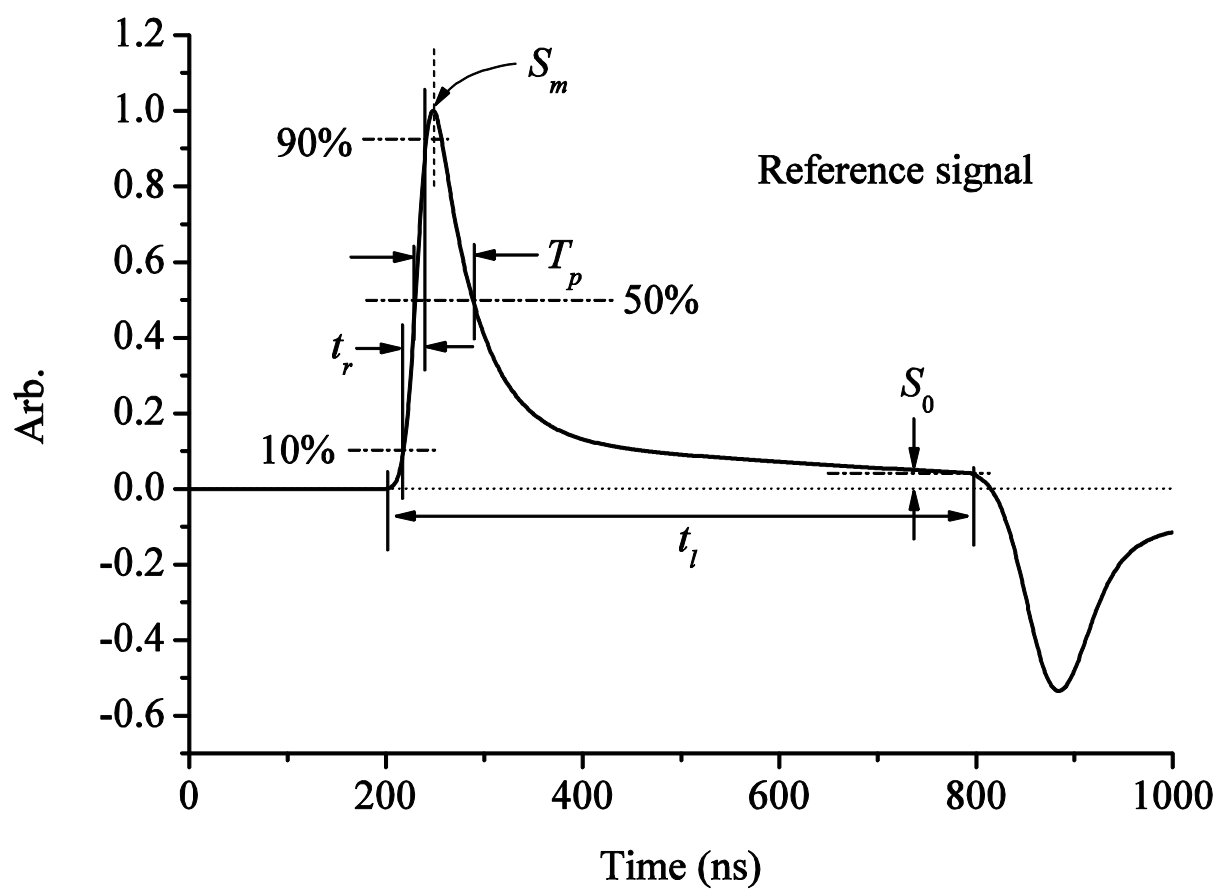




\section{Figure 6.}

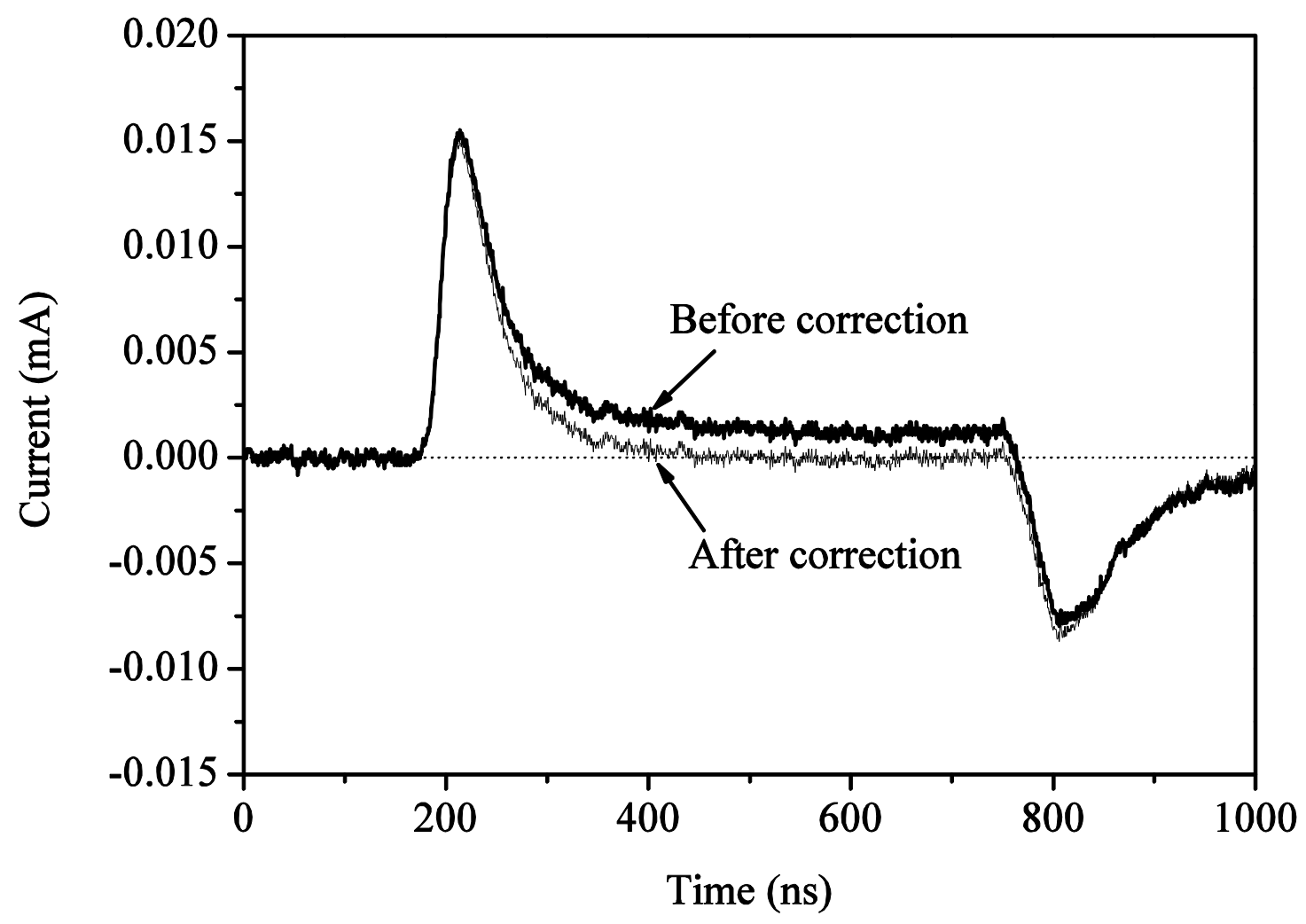


Figure 7.

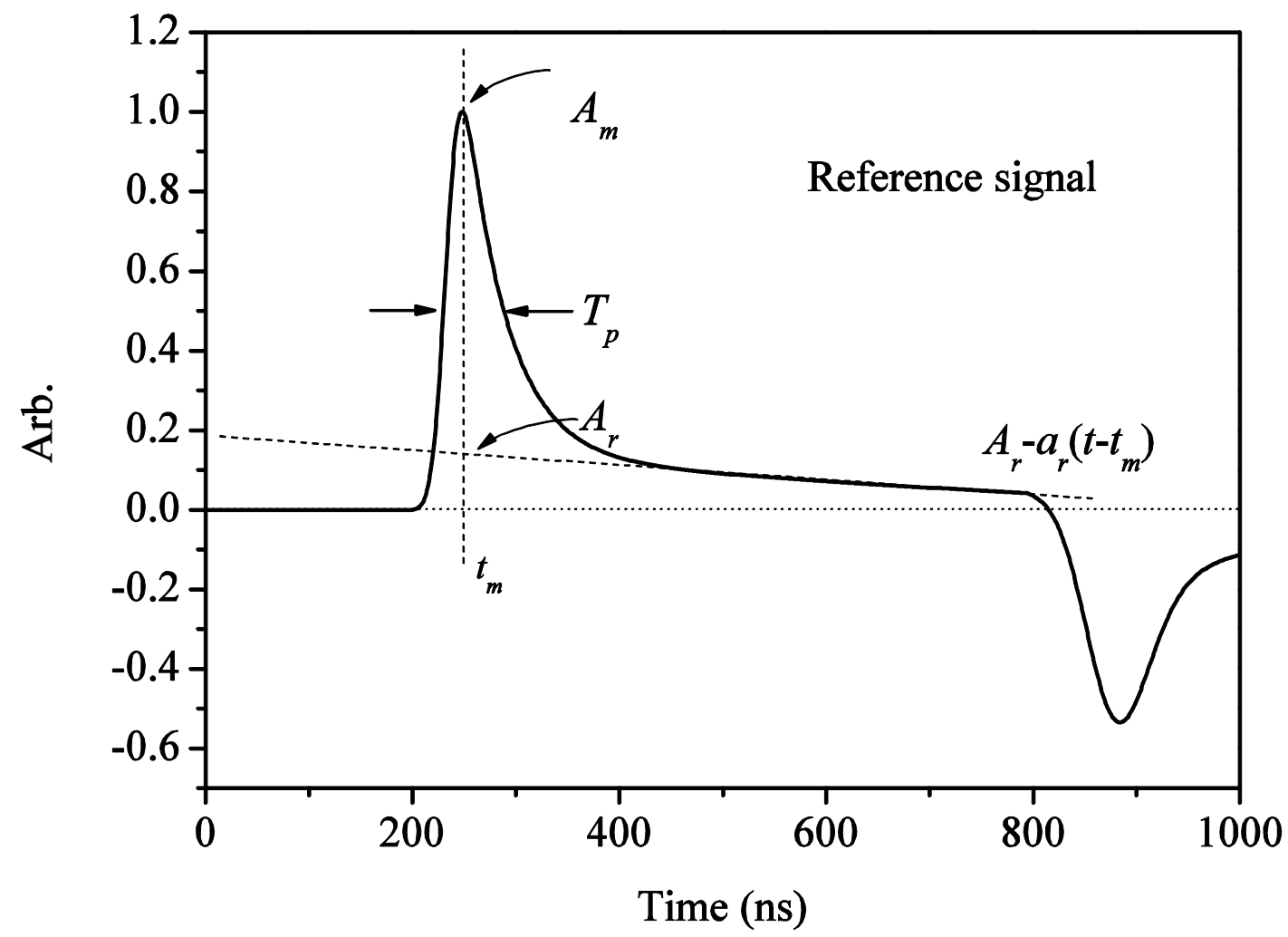




\section{Figure 8.}

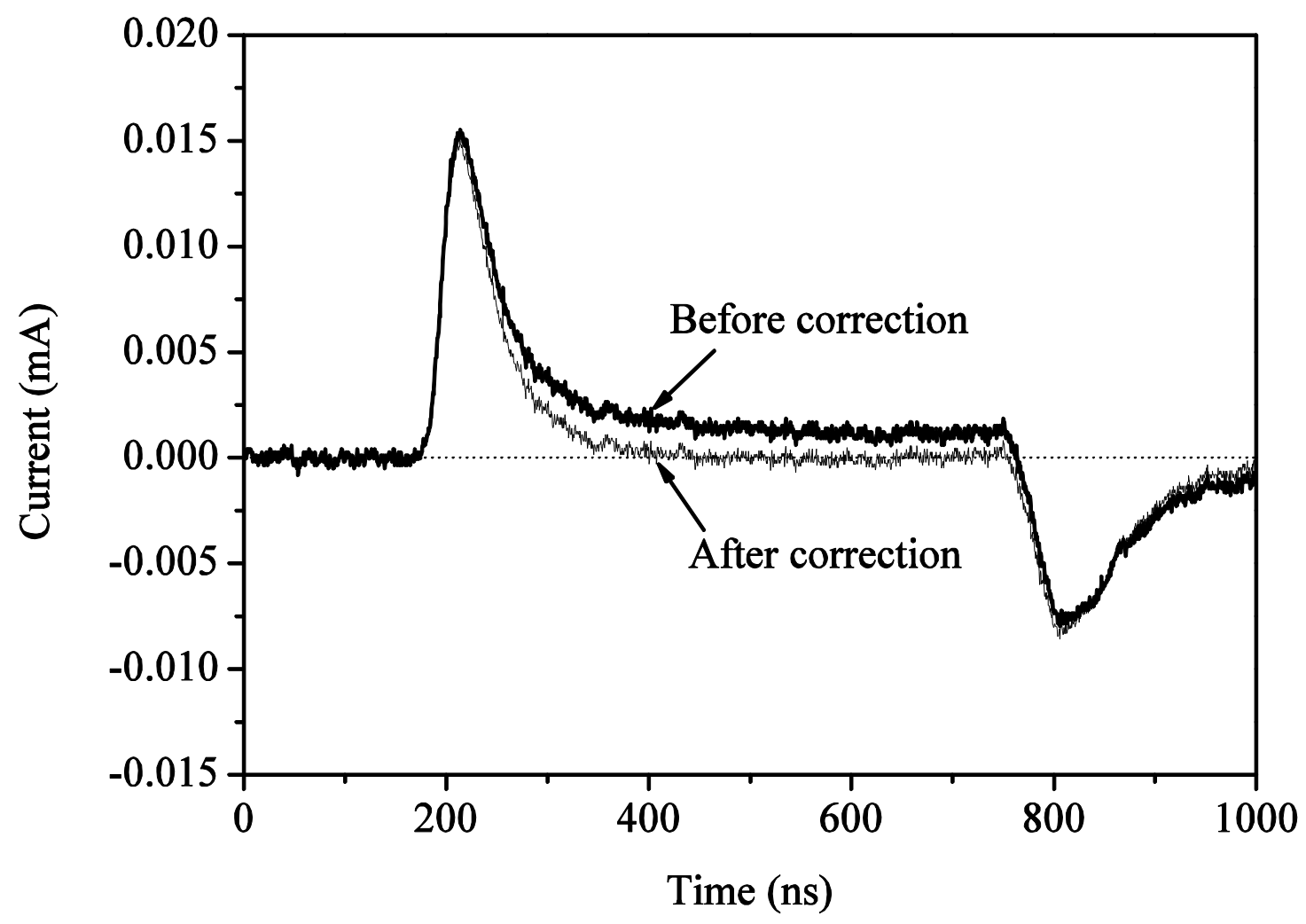




\section{Figure 9.}

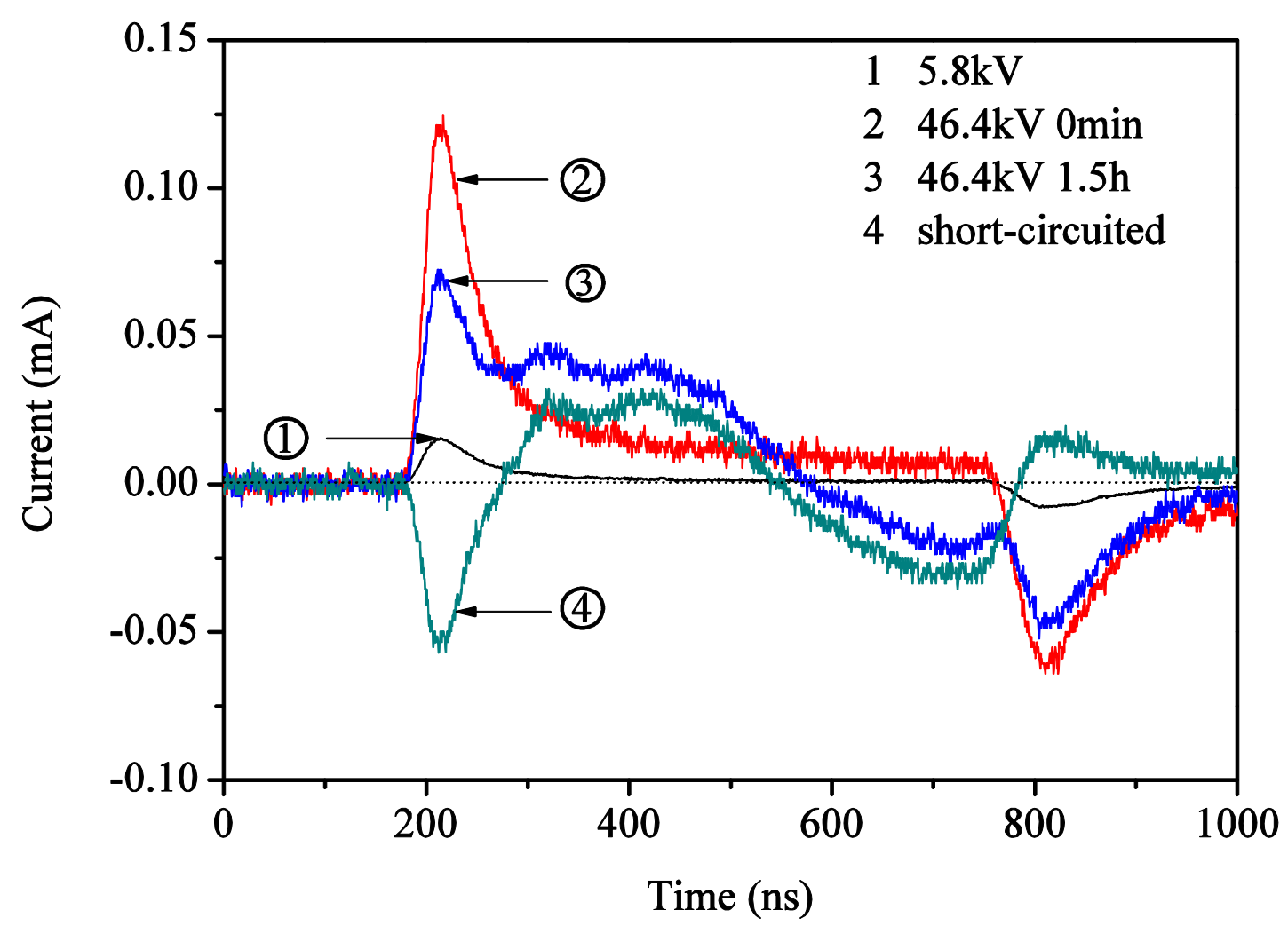




\section{Figure 10.}
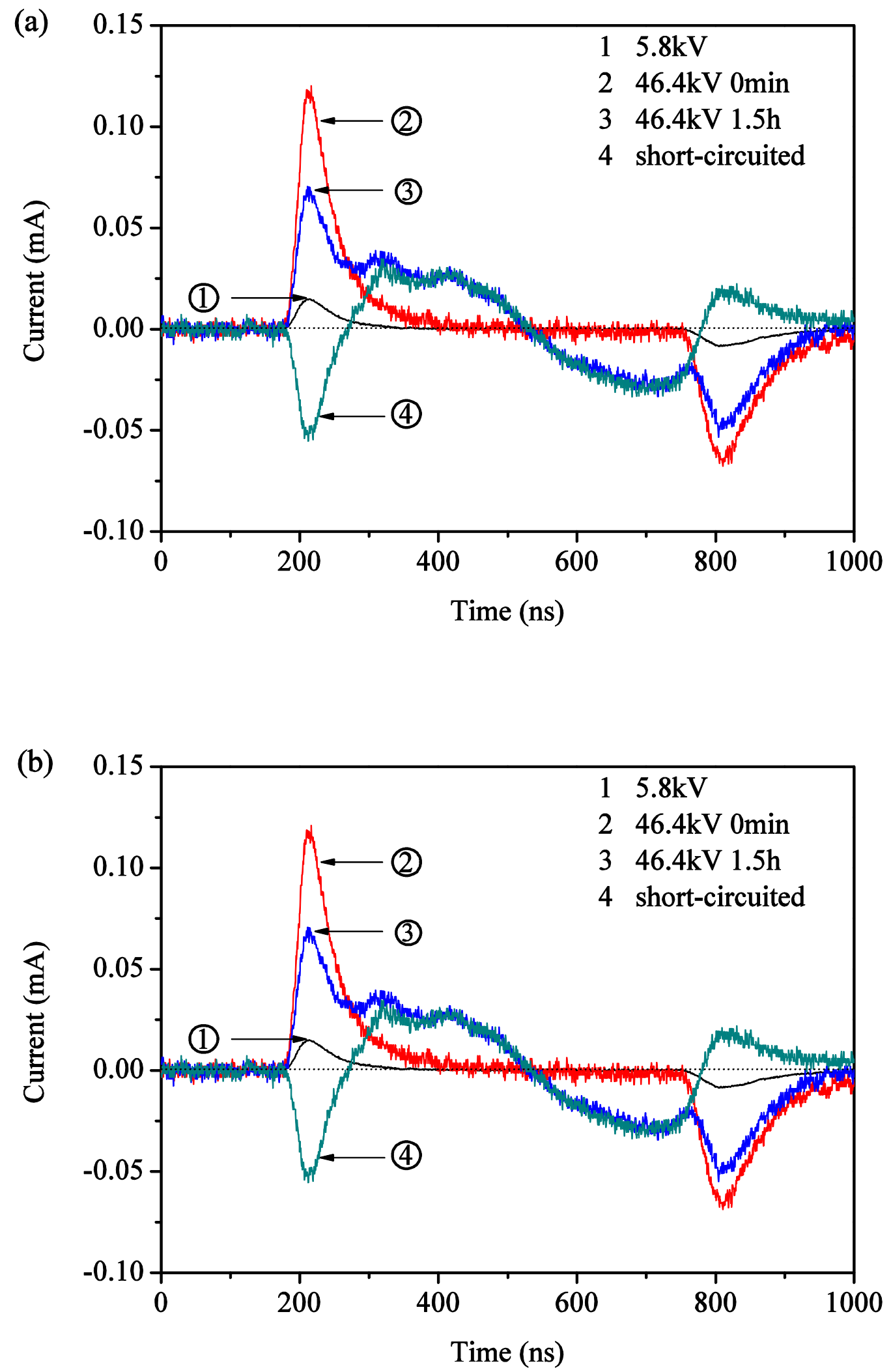


\section{Figure 11.}

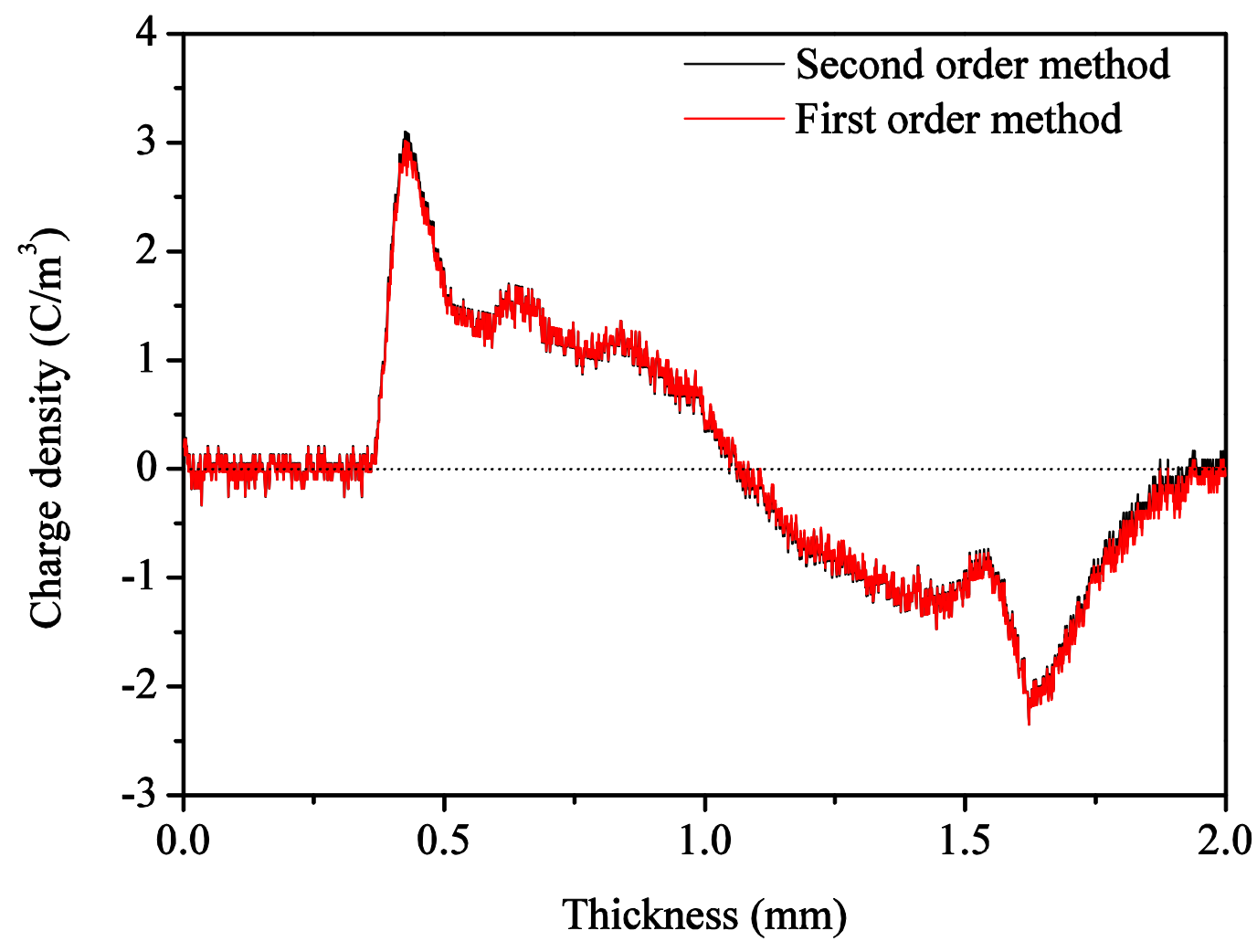




\section{Figure 12.}

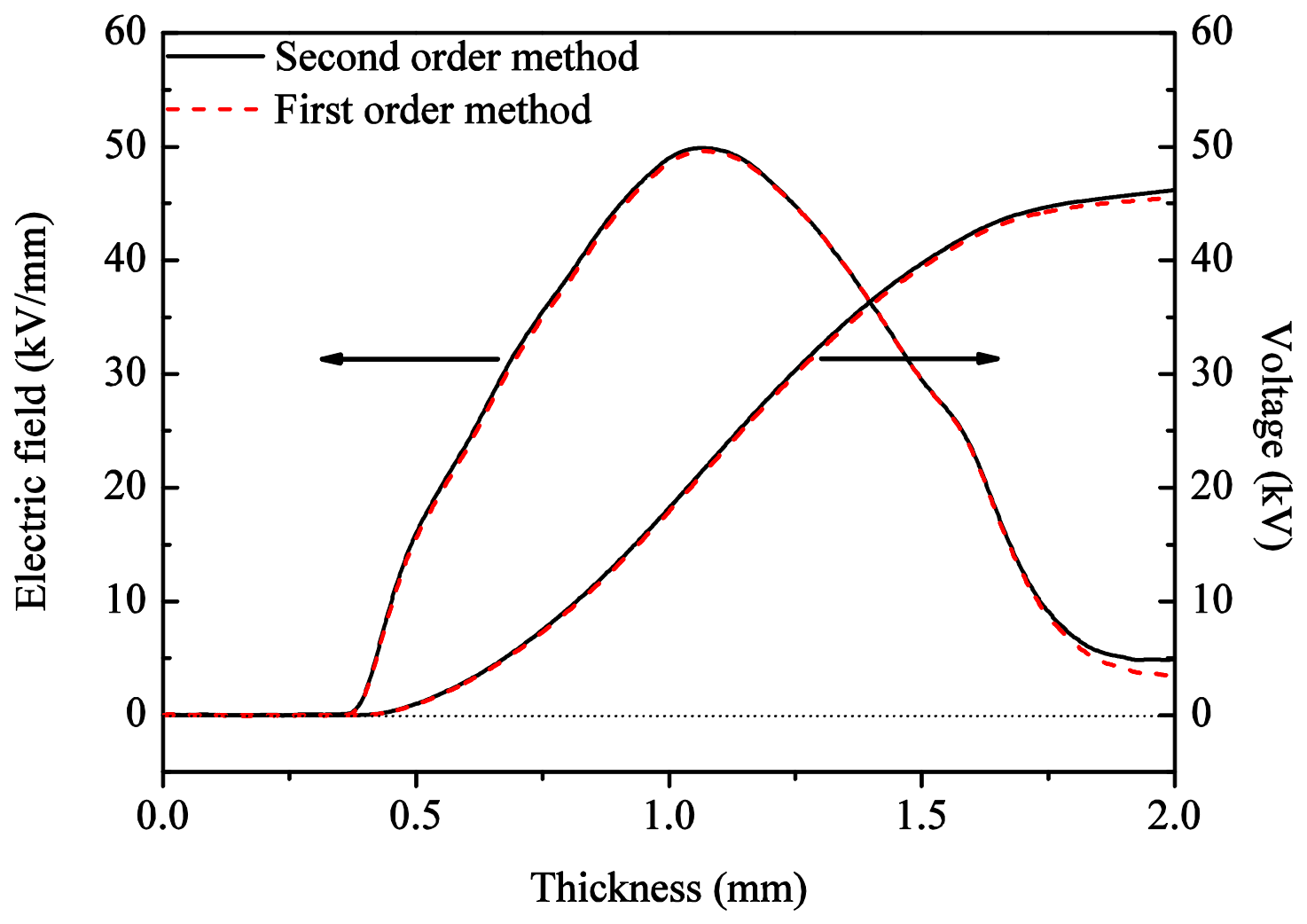

